\title{
Generation and detection of bound entanglement
}

\author{
P. Hyllus ${ }^{1}$, C. Moura Alves ${ }^{2,3}$, D. Bruß ${ }^{1}$, and C. Macchiavello ${ }^{4}$ \\ ${ }^{1}$ Institut für Theoretische Physik, Universität Hannover, 30167 Hannover, Germany \\ ${ }^{2}$ Clarendon Laboratory, University of Oxford, Parks Road, Oxford OX1 3PU, UK \\ ${ }^{3}$ Centre for Quantum Computation, DAMTP, University of Cambridge, Wilberforce Road, Cambridge CB3 0WA, UK \\ ${ }^{4}$ Dipartimento di Fisica "A. Volta" and INFM-Unitá di Pavia, Via Bassi 6, 27100 Pavia, Italy
}

(Dated: October 8, 2018)

\begin{abstract}
We propose a method for the experimental generation of two different families of bound entangled states of three qubits. Our method is based on the explicit construction of a quantum network that produces a purification of the desired state. We also suggest a route for the experimental detection of bound entanglement, by employing a witness operator plus a test of the positivity of the partial transposes.
\end{abstract}

PACS numbers: 03.67.-a, 03.65.Ud, 03.67.Mn

\section{INTRODUCTION}

Entanglement, one of the central themes in quantum information processing, is well understood in low-dimensional systems. In dimensions $2 \times 2$ and $2 \times 3$, a necessary and sufficient condition for entanglement exists: the partial transposition test [1, 2]. However, the properties of entanglement are much less clear in higher-dimensional systems, for which only sufficient conditions for a density matrix to be entangled are known [3, 4, 5]. There exist higherdimensional states that, although entangled, have a positive partial transpose (PPT). Due to this property, it is not possible to distill any entanglement from them with local operations and classical communication (LOCC). Undistillable states are also called bound entangled []. For systems consisting of more than two parties, a state may be undistillable even if some of the partial transposes are non-positive [7]. Even for bipartite systems, bound entangled states with non-positive partial transpose probably exist [8].

Apart from the interesting fundamental nature of bound entangled states, their usefulness for quantum information processing has been studied: bound entangled states can activate the distillability of one copy of a bipartite state with non-positive partial transpose [9, 10]. It has also recently been shown that one can extract a secure key from bound entangled states [11. In the context of key creation, results from quantum information theory, with special use of bound entangled states, have recently been proven to be fruitful for insights into open classical information theoretical issues [12]. Various classes of entangled states have been constructed theoretically [13]. However, the topic of generating bound entanglement in the laboratory and proving the produced state to be bound entangled has not been addressed so far.

How does one generate a certain bound entangled state experimentally? A solution that is straightforward from a theoretical point of view is to consider the spectral decomposition of the state and to compose a mixed density matrix by creating the eigenvectors with probabilities that are specified by the according eigenvalues. However, this is, in general, a demanding experimental task, as one would need a source that can emit various types of product vectors and entangled vectors with high fidelities and well-specified probabilities. A more satisfactory approach is to deterministically generate a state that is the purification of the wanted bound entangled state in some higherdimensional Hilbert space. The additional dimensions are provided by ancilla systems. Then, by tracing out the ancilla (i.e. experimentally simply ignoring the ancilla part), one arrives at the desired bound entangled state.

In this paper we develop the latter method. Namely, we explicitly construct quantum networks that generate the two families of bound entangled states of three qubits introduced in [14] and [7]. The first family is PPT with respect to any of the subsystems but nevertheless entangled, while the second family has a parameter range in which it is NPT only with respect to one subsystem which is not sufficient for distillation of a singlet between any two of the parties 7]. The properties of the latter states have been used recently in the context of quantum cryptography to show that so-called bound information exists [12. The networks in both cases act on a six-qubit register that is initially in state $|000000\rangle$, and from which they generate a six-qubit pure state, such that the reduced density operator $\rho_{\text {bound }}$ of the first three qubits is the desired bound entangled state.

The network for the family 14 requires only eight two-qubit gates and one Toffoli gate with three control qubits, while the network for the family [7] requires six CNOT gates, one control-U with two control qubits and one Toffoli gate with three control qubits. The number of qubits and number of gates is in foreseeable reach of quantum information technology: at present, with NMR techniques an order-finding algorithm has been performed with 5 qubits and 6 control gates [15]. In ion traps, 6 qubits could be provided, and control gates and simple algorithms have been 
demonstrated [16].

The second step for the experimental generation of bound entangled states is to show that the generated states indeed carry bound entanglement. For the family of bound entangled states in 14 we discuss this issue explicitly. The entanglement of the state can be proved by using an entanglement witness [5]. We construct an appropriate witness, and provide its local decomposition which requires only four measurements settings. Furthermore, this family of states has a PPT with respect to any subsystem. For the experimental proof of this fact we compare three methods: we consider the full state estimation of the produced state $\rho_{\text {bound }}$, the more direct spectrum estimation of $\rho_{\text {bound }}^{\prime}=S P A\left(\rho_{\text {bound }}\right)$, where $S P A$ is the LOCC version of the structural physical approximation to the partial transpose [17], and finally the spectrum estimation of the partial transpose of $\rho_{\text {bound }}$ via the LOCC version of the network introduced in $[18$.

The paper is organized as follows. In Sec. 2 we will introduce the network that generates the class of bound entangled states described in [14]. In Sec. 3 we construct the entanglement witness that detects entanglement in the density matrix. In Sec. 4 we discuss the three different approaches to check the positivity of the partial transpositions of the density matrix with respect to any of the three subsystems. In Sec. 5, we construct a network that generates the family of bound entangled states of Ref. 7] and discuss how the methods applied in Secs. 3 and 4 could be used to experimentally prove the existence of bound entanglement in this case. Finally, in Sec. 6 we conclude with a summary of our results.

\section{GENERATION OF BOUND ENTANGLED STATES}

In this section we explicitly construct the quantum network that generates the following class of bound entangled states [14]:

$\rho_{\text {bound }}=\frac{1}{N}\left(2|G H Z\rangle\langle G H Z|+a| 001\rangle\langle 001|+b| 010\rangle\langle 010|+c| 011\rangle\left\langle 011\left|+\frac{1}{c}\right| 100\right\rangle\left\langle 100\left|+\frac{1}{b}\right| 101\right\rangle\left\langle 101\left|+\frac{1}{a}\right| 110\right\rangle\langle 110|\right)$,

where $|G H Z\rangle=(|000\rangle+|111\rangle) / \sqrt{2}$, the coefficients fulfill $a, b, c>0$ and $a b \neq c$, while the normalization reads $N=2+a+b+c+1 / a+1 / b+1 / c$. This mixed state can be generated deterministically by a quantum network that uses a register with three qubits plus three auxiliary qubits, all initialized at $|0\rangle$, and generates a pure states of 6 qubits, such that the reduced density operator of the three qubits of interest is $\rho_{\text {bound }}$.

The procedure to generate the bound entangled state consists of two parts: a preparation stage for the first three qubits, and a purification stage where from the prepared state and an ancilla state a purification of $\rho_{\text {bound }}$ is generated. In the preparation stage one starts with the three-qubit state $|000\rangle$, and prepares a three-qubit pure state of the form

$$
\left|\psi_{\text {bound }}\right\rangle=\frac{1}{\sqrt{N}}\left(|000\rangle+\sqrt{a}|001\rangle+\sqrt{b}|010\rangle+\sqrt{c}|011\rangle+\frac{1}{\sqrt{c}}|100\rangle+\frac{1}{\sqrt{b}}|101\rangle+\frac{1}{\sqrt{a}}|110\rangle+|111\rangle\right) .
$$

This is achieved by applying certain local rotations (LU) on the three qubits, a control-U gate $\mathrm{CU}_{(3,1)}$ between qubit 3 and qubit 1 (qubit 3 acts as the control qubit), a control- $U$ gate $\mathrm{CU}_{(3,2)}$ between qubit 3 and qubit 2 (qubit 3 acts as the control qubit) and a CNOT gate between qubit 1 and qubit 3 (qubit 1 acts as the control qubit). This sequence of gates is illustrated in the left part of Fig. 1]

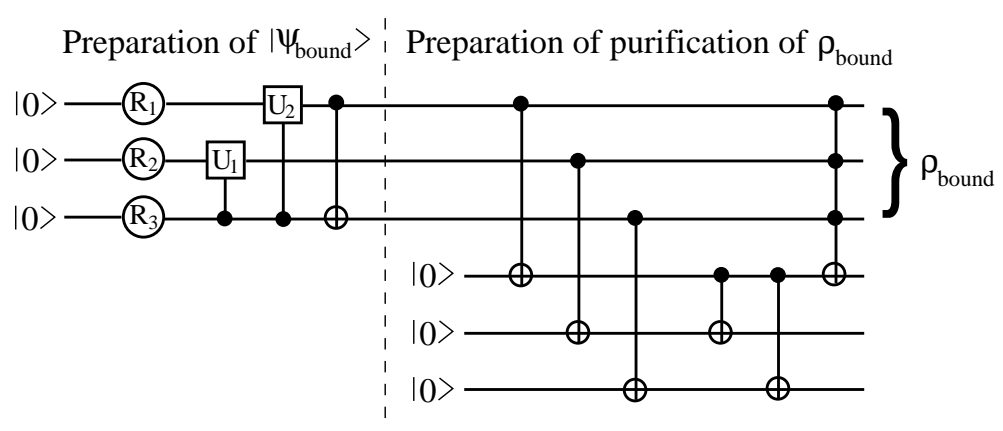

FIG. 1: The network for creating the bound entangled state given in Eq. (1). 
The specific form of these gates is given by

$$
\begin{aligned}
\mathrm{LU} & =N_{1}\left(\begin{array}{cc}
1 & 1 / \sqrt{b} \\
1 / \sqrt{b} & -1
\end{array}\right)_{1} \otimes N_{2}\left(\begin{array}{cc}
1 & \sqrt{b} \\
\sqrt{b} & -1
\end{array}\right)_{2} \otimes\left(\begin{array}{cc}
\alpha & \beta \\
\beta & -\alpha
\end{array}\right)_{3} \\
\mathrm{CU}_{(3,1)} & =\mathbb{I}_{(1,2)} \otimes|0\rangle\left\langle\left. 0\right|_{3}+N_{1} N_{3}\left(\begin{array}{ll}
(\sqrt{a}-\sqrt{1 / b c}) & (\sqrt{a / b}+\sqrt{1 / c}) \\
(\sqrt{a / b}+\sqrt{1 / c}) & (-\sqrt{a}+\sqrt{1 / b c})
\end{array}\right)_{1} \otimes \mathbb{I}_{2} \otimes \mid 1\right\rangle\left\langle\left. 1\right|_{3},\right. \\
\mathrm{CU}_{(3,2)} & =\mathbb{I}_{(1,2)} \otimes|0\rangle\left\langle\left. 0\right|_{3}+\mathbb{I}_{1} \otimes N_{2} N_{4}\left(\begin{array}{cc}
(1-\sqrt{b c / a}) & (\sqrt{b}+\sqrt{c / a}) \\
(\sqrt{b}+\sqrt{c / a}) & (-1+\sqrt{b c / a})
\end{array}\right)_{2} \otimes \mid 1\right\rangle\left\langle\left. 1\right|_{3} .\right.
\end{aligned}
$$

where $N_{1}=\sqrt{b /(1+b)}, N_{2}=1 / \sqrt{1+b}, N_{3}=\sqrt{c /(1+a c)}$, and $N_{4}=\sqrt{a /(a+c)}$. The coefficients $\alpha$ and $\beta$ depend on $a, b, c$ and must be chosen such that $\alpha N_{1} N_{2}=\beta N_{3} N_{4}$ and $\alpha^{2}+\beta^{2}=1$.

It is straightforward to confirm that this set of gates is constructed such that it performs the following sequence of transformations:

$$
\begin{aligned}
& |000\rangle \quad \stackrel{\mathrm{LU}}{\longrightarrow} \quad N_{1}\left(|0\rangle+\frac{1}{\sqrt{b}}|1\rangle\right) N_{2}(|0\rangle+\sqrt{b}|1\rangle)(\alpha|0\rangle+\beta|1\rangle) \\
& \stackrel{\mathrm{CU}_{(3,1)} \cdot \mathrm{CU}_{(3,2)}}{\longrightarrow} \frac{1}{\sqrt{N}}\left[\left(|0\rangle+\frac{1}{\sqrt{b}}|1\rangle\right)(|0\rangle+\sqrt{b}|1\rangle)|0\rangle+\left(\sqrt{a}|0\rangle+\frac{1}{\sqrt{c}}|1\rangle\right)\left(|0\rangle+\sqrt{\frac{c}{a}}|1\rangle\right)|1\rangle\right] \\
& \stackrel{\mathrm{CNOT}_{(1,3)}}{\longrightarrow}\left|\psi_{\text {bound }}\right\rangle \text {. }
\end{aligned}
$$

In the second part of the network one first applies a sequence of three CNOT gates between the main and the auxiliary qubits: in this way each term of $\left|\psi_{\text {bound }}\right\rangle$ is copied to the ancilla system. Here, the first, second and third qubits of the main system act as control qubits, and the first, second and third ancilla qubits act as target qubits, respectively:

$$
\begin{aligned}
\left|\psi_{\text {bound }}\right\rangle|000\rangle \stackrel{\text { CNOTs }}{\longrightarrow} \frac{1}{\sqrt{N}}(|000\rangle|000\rangle & +\sqrt{a}|001\rangle|001\rangle+\sqrt{b}|010\rangle|010\rangle+\sqrt{c}|011\rangle|011\rangle+\frac{1}{\sqrt{c}}|100\rangle|100\rangle \\
& \left.+\frac{1}{\sqrt{c}}|101\rangle|101\rangle+\frac{1}{\sqrt{a}}|110\rangle|110\rangle+|111\rangle|111\rangle\right) .
\end{aligned}
$$

Applying $\mathrm{CNOT}_{(4,5)}$ and $\mathrm{CNOT}_{(4,6)}$ then leads to

$$
\begin{aligned}
\longrightarrow & \frac{1}{\sqrt{N}}(|000\rangle|000\rangle+\sqrt{a}|001\rangle|001\rangle+\sqrt{b}|010\rangle|010\rangle+\sqrt{c}|011\rangle|011\rangle \\
& \left.+\frac{1}{\sqrt{c}}|100\rangle|111\rangle+\frac{1}{\sqrt{c}}|101\rangle|110\rangle+\frac{1}{\sqrt{a}}|110\rangle|101\rangle+|111\rangle|100\rangle\right) .
\end{aligned}
$$

Finally, one applies a 3-Toffoli gate, where the three system qubits are the control qubits and the first auxiliary qubit is the target. Its action is defined as [19]

$$
|a, b, c\rangle|f\rangle \rightarrow|a, b, c\rangle|a \cdot b \cdot c \oplus f\rangle .
$$

The resulting state of the total system is then

$$
\begin{aligned}
\left|\Psi_{\text {bound }}\right\rangle=\frac{1}{\sqrt{N}}((|000\rangle+|111\rangle)|000\rangle & +\sqrt{a}|001\rangle|001\rangle+\sqrt{b}|010\rangle|010\rangle+\sqrt{c}|011\rangle|011\rangle+\frac{1}{\sqrt{c}}|100\rangle|111\rangle \\
& \left.+\frac{1}{\sqrt{b}}|101\rangle|110\rangle+\frac{1}{\sqrt{a}}|110\rangle|101\rangle\right) .
\end{aligned}
$$

Tracing over the three auxiliary qubits, one obtains that the remaining state of the three system qubits is of the desired form of Eq. (11):

$$
\begin{aligned}
\operatorname{Tr}_{\text {aux }}\left(\left|\Psi_{\text {bound }}\right\rangle\left\langle\Psi_{\text {bound }}\right|\right)=\frac{1}{N}(2|G H Z\rangle\langle G H Z| & +a|001\rangle\langle 001|+b| 010\rangle\langle 010|+c| 011\rangle\langle 011| \\
& \left.+\frac{1}{c}|100\rangle\left\langle 100\left|+\frac{1}{b}\right| 101\right\rangle\left\langle 101\left|+\frac{1}{a}\right| 110\right\rangle\langle 110|\right)=\rho_{\text {bound }}
\end{aligned}
$$


The total quantum network that generates the bound entangled state $\rho_{\text {bound }}$ is shown in Fig. 1 .

Note that for the generation of this bound entangled state a more general version of the Toffoli gate can also be applied, namely $|a, b, c\rangle|f\rangle \rightarrow \exp [i \theta(a, b, c)]|a, b, c\rangle|a \cdot b \cdot c \oplus f\rangle$, because the extra phases cancel when one traces over the ancilla qubits after the Toffoli gate. This requires less elementary operations than the Toffoli gate 19]. The Toffoli gate with three controls can be decomposed into 13 two-qubit gates [20]. We point out that in this paper we are mainly interested in providing a network for the generation of bound entanglement with a small number of gates, rather than in the optimization of this network, or the decomposition of the necessary gates into elementary single and two-qubit gates. The latter issue is discussed elsewhere in the literature [21].

\section{CONSTRUCTION AND DECOMPOSITION OF THE ENTANGLEMENT WITNESS}

In this section we will construct and locally decompose an entanglement witness $W$ that allows to detect the entanglement of $\rho_{\text {bound }}$ with only four local measurements. An entanglement witness [4, 5] is an operator with nonnegative expectation value on separable states, and with negative expectation value on some entangled states. Thus, if we construct an appropriate witness for $\rho_{\text {bound }}$ and then measure its expectation value, the experimental result

$$
\operatorname{Tr}\left(W \rho_{\text {bound }}\right)<0
$$

indicates that $\rho_{\text {bound }}$ is entangled. Witnesses are observables with non-local eigenvectors which would be difficult to measure directly. However, witness operators can be decomposed locally [22] and thus be easily measured in an experiment.

The state $\rho_{\text {bound }}$ that we want to detect has a positive partial transpose with respect to every subsystem, but there is no product vector $|\phi\rangle$ in its range s.t. $\left|\phi^{* X}\right\rangle$ is in the range of $\rho_{\text {bound }}^{T_{X}}$ (here $X=A, B, C$; the symbol $T_{X}$ denotes partial transposition with respect to subsystem $X$, and ${ }^{*} X$ denotes complex conjugation with respect to subsystem $X$ ). Such states are called bound entangled edge states [5]. Any PPT entangled state cannot be detected by decomposable witnesses, i.e. witnesses which are of the form $W=P+Q^{T_{X}}$, where $P$ and $Q$ are positive operators. However, there are methods for constructing witnesses that detect bound entangled edge states. We will follow the methods of Ref. 5] for the construction of our witness [23], namely:

$$
W=\bar{W}-\epsilon \mathbb{I},
$$

where

$$
\bar{W}=P+Q_{A}^{T_{A}}+Q_{B}^{T_{B}}+Q_{C}^{T_{C}}
$$

where $P$ denotes the projector onto the kernel of $\rho_{B}$ and $Q_{X}$ is the projector onto the kernel of $\rho_{B}^{T_{X}}$. The parameter $\epsilon$ is given by

$$
\epsilon=\inf _{|e, f, g\rangle}\langle e, f, g|\bar{W}| e, f, g\rangle,
$$

from which $\epsilon>0$ follows [5]. We find that

$$
\begin{aligned}
\bar{W}= & \frac{1}{2}(|000\rangle\langle 000|+| 111\rangle\langle 111|)+\frac{1}{1+c^{2}}\left(c^{2}|100\rangle\langle 100|+| 011\rangle\langle 011|\right)+\frac{1}{1+b^{2}}\left(|010\rangle\left\langle 010\left|+b^{2}\right| 101\right\rangle\langle 101|\right) \\
& +\frac{1}{1+a^{2}}\left(|001\rangle\left\langle 001\left|+a^{2}\right| 110\right\rangle\langle 110|\right)-\left[\frac{1}{2}+\frac{c}{1+c^{2}}+\frac{b}{1+b^{2}}+\frac{a}{1+a^{2}}\right](|000\rangle\langle 111|+| 111\rangle\langle 000|) . \quad(15)
\end{aligned}
$$

Employing the Pauli operators $\sigma_{z}=|0\rangle\langle 0|-| 1\rangle\left\langle 1\left|, \sigma_{x}=\right| 0\right\rangle\langle 1|+| 1\rangle\langle 0|$, and $\sigma_{y}=-i|0\rangle\langle 1|+i| 1\rangle\langle 0|$, one can use the decomposition [24]

$$
|000\rangle\langle 111|+| 111\rangle\langle 000|=\frac{1}{4}\left(\sigma_{x}^{\otimes 3}-\sigma_{x} \sigma_{y} \sigma_{y}-\sigma_{y} \sigma_{x} \sigma_{y}-\sigma_{y} \sigma_{y} \sigma_{x}\right)=\frac{1}{2}\left(\sigma_{x}^{\otimes 3}-\frac{1}{4}\left(\sigma_{x}+\sigma_{y}\right)^{\otimes 3}-\frac{1}{4}\left(\sigma_{x}-\sigma_{y}\right)^{\otimes 3}\right) .
$$

For the last expression the local operators $\sigma_{x}^{\otimes 3}$ and $\left(\left(\sigma_{x} \pm \sigma_{y}\right) / \sqrt{2}\right)^{\otimes 3}$ have to be measured. All the other projectors in Eq. (15) can be measured with a single $\sigma_{z}^{\otimes 3}$ measurement. Hence, the measurement of the witness requires only 4 measurement settings. Using the methods of [24] this number can be proved to be optimal. On the other hand, if state 


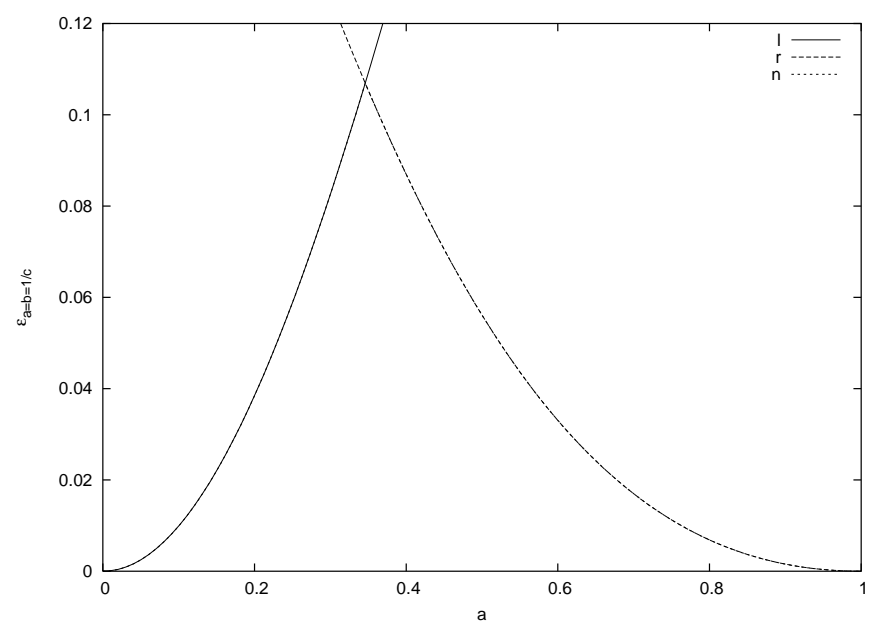

FIG. 2: Lower bound for $\epsilon_{a=b=1 / c}$ as a function of $a$ : The left curve (l) is given by $a^{2} /\left(1+a^{2}\right)$, while the right curve (r) is the analytic minimum of $\epsilon$ for $\theta_{e}=\theta_{f}=\theta_{g}$. The maximal lower bound $\epsilon^{\text {max }} \geq 0.1069$ is obtained for $a=0.3460$, where the two curves meet. The result of the numerical minimization (n) is plotted on top of the two analytical curves, and equals the lower branch of them for all $a$.

tomography is applied to confirm the positivity of the partial transposes (c.f. chapter IV.), then all measurements necessary for the witness with the first decomposition of Eq. (16) are already performed there.

The last step on the construction of our witness is the computation of the value of $\epsilon$. We use the parametrization $|e\rangle=\cos \theta_{e}|0\rangle+\exp i \phi_{e} \sin \theta_{e}|1\rangle$ and accordingly for $|f\rangle$ and $|g\rangle$. This leads to

$$
\begin{aligned}
\epsilon= & \inf _{|e, f, g\rangle}\left[\frac{1}{2}\left(\left(c_{e} c_{f} c_{g}\right)^{2}+\left(s_{e} s_{f} s_{g}\right)^{2}\right)+\frac{1}{1+c^{2}}\left(c^{2}\left(s_{e} c_{f} c_{g}\right)^{2}+\left(c_{e} s_{f} s_{g}\right)^{2}\right)+\frac{1}{1+b^{2}}\left(\left(c_{e} s_{f} c_{g}\right)^{2}+b^{2}\left(s_{e} c_{f} s_{g}\right)^{2}\right)\right. \\
& \left.+\frac{1}{1+a^{2}}\left(\left(c_{e} c_{f} s_{g}\right)^{2}+a^{2}\left(s_{e} s_{f} c_{g}\right)^{2}\right)-\left[\frac{1}{2}+\frac{c}{1+c^{2}}+\frac{b}{1+b^{2}}+\frac{a}{1+a^{2}}\right]\left(2 \cos \left(\phi_{e}+\phi_{f}+\phi_{g}\right) c_{e} c_{f} c_{g} s_{e} s_{f} s_{g}\right)\right],
\end{aligned}
$$

where $c_{e, f, g} \equiv \cos \theta_{e, f, g}$ and $s_{e, f, g} \equiv \sin \theta_{e, f, g}$. In this equation the phases $\phi_{e}, \phi_{f}, \phi_{g}$ appear only in the term $\cos \left(\phi_{e}+\phi_{f}+\phi_{g}\right)$. Therefore the phases can be chosen to be equal to zero, using the following argument: the term $\left(2 \cos \left(\phi_{e}+\phi_{f}+\phi_{g}\right) c_{e} c_{f} c_{g} s_{e} s_{f} s_{g}\right)$ in the above equation has to have a positive sign in order to minimize $\epsilon$. As the coefficients $c_{e, f, g}$ and $s_{e, f, g}$ occur only quadratically in all other terms, all of them can be chosen to be positive. Then $\epsilon$ is minimized for $\phi_{e}=\phi_{f}=\phi_{g}=0$. We are thus left with 6 real parameters. If the parameters $a, b, c$ are determined by the experimental set-up, then the corresponding value of $\epsilon$ can be obtained numerically by use of a multivariable minimization routine.

If the parameters $a, b, c$ can be chosen freely, then it is advantageous to maximize $\epsilon$ with respect to $a, b, c$. Making the natural assumption that white noise is introduced in the preparation procedure of the state, i.e. $\rho_{p}=p \rho_{\mathrm{B}}+\frac{1-p}{8} \mathbb{I}$, the witness will detect entanglement in the state for $p>1-2 \epsilon$. Hence the tolerance of the witness to the presence of noise is enlarged by maximizing $\epsilon$. We searched for the maximum in the parameter range $a=b=1 / c \in] 0,1[$. (Remember from the definition of $\rho_{\text {bound }}$ in Eq. (11) that one has to use the open interval here.) We obtain numerically that for $a<a_{\mathrm{th}}=0.3460$ the minimum is reached at $\epsilon=a^{2} /\left(1+a^{2}\right)$, i.e. when the product state is one of the three possibilities $|e, f, g\rangle=|011\rangle,|101\rangle,|110\rangle$. For $a>a_{\mathrm{th}}$ the minimum of $\epsilon$ is obtained when $\theta_{e}=\theta_{f}=\theta_{g}$. These results are shown in Fig. 22 We find $\epsilon_{a=b=1 / c}^{\max } \geq 0.1069$ which is reached for $a_{\mathrm{th}}=0.3460$. This is also the highest value obtained numerically when $a, b, 1 / c \in] 0,1[$ without the restriction $a=b=1 / c$. For this choice of parameters the state mixed with white noise as described above is still detected for $p>0.786$, i.e. more than $20 \%$ of white noise can be tolerated.

\section{TESTING THE POSITIVITY OF THE PARTIAL TRANSPOSE}

In this section we present three different methods to check the positivity of the partially transposed density operator $\rho_{\text {bound }}^{T_{X}}$ with respect to subsystem $X=A, B, C$. One possible option is to perform the full state estimation of $\rho_{\text {bound }}$ [25], and then to check whether all the eigenvalues of $\rho_{\text {bound }}^{T_{X}}$ for $X=A, B, C$ are positive. This method 
requires the estimation of $(2 \times 2 \times 2)^{2}-1=63$ independent parameters of the density operator. This can be achieved by performing $3 \times 3 \times 3=27$ measurements on single copies of $\rho_{\text {bound }}$, since one can write any three qubit state as

$$
\rho=\frac{1}{8} \sum_{i, j, k=0, x, y, z} \lambda_{i, j, k} \sigma_{i} \otimes \sigma_{j} \otimes \sigma_{k},
$$

where $\lambda_{i, j, k}=\operatorname{tr}\left(\rho \sigma_{i} \otimes \sigma_{j} \otimes \sigma_{k}\right)$, and $\sigma_{0}=\mathbb{I}$. The data from estimating $\lambda_{l, m, n}$ with $l, m, n=x, y, z$ can also be used to estimate $\lambda_{0, m, n}, \lambda_{l, 0, n}$ and $\lambda_{l, m, 0}$. Hence, only local measurements in the $x, y, z$ directions have to be performed. One disadvantage of this option is the superfluous estimation of parameters of the density operator, since we are only interested in learning about the lowest eigenvalue of the partially transposed density operator.

Another method for finding out whether $\rho_{\text {bound }}^{T_{X}}>0$ for $X=A, B, C$ is to start by applying the structural physical approximation (SPA) 17] to the partial transpose of $\rho_{\text {bound, }}$, and then to estimate the lowest eigenvalue of the resulting density operator. A structural physical approximation is a completely positive (CP) map, constructed from a positive, but not CP map, by adding white noise. The aim in constructing these approximations is to allow the physical implementation of maps which are useful in entanglement detection, but are non-physical. In this way one is able to bypass full state estimation when trying to detect the existence of entanglement in a given system, since one can estimate directly the relevant parameters, e.g the lowest eigenvalue. The construction of the SPA for a positive, but not completely positive map $\Lambda$, is as follows:

$$
\widetilde{[\mathbb{I} \otimes \Lambda}](\rho)=\frac{d^{4} \lambda}{d^{4} \lambda+1} \frac{I \otimes I}{d^{2}}+\left(1-\frac{d^{4} \lambda}{d^{4} \lambda+1}\right)[\mathbb{I} \otimes \Lambda](\rho),
$$

where $d$ is the dimension of each of the two subsystems on which $[\mathbb{I} \otimes \Lambda]$ acts, and $\lambda$ is the absolute value of the most negative eigenvalue obtained when $[(\mathbb{I} \otimes \mathbb{I})(\mathbb{I} \otimes \Lambda)]$ acts on the maximally entangled state $\sum_{i=1}^{d^{2}}|i\rangle|i\rangle / \sqrt{d^{2}}$. Each state $|i\rangle$ pertains to a $d^{2}$-dimensional system, itself composed of two subsystems of dimension $d$. If one takes $\Lambda$ to be the transposition map $T$, one finds $\lambda=1 / d$. In the two-qubit case, one obtains that

$$
\widetilde{[\mathbb{I} \otimes T}](\rho)=\frac{2}{9} I \otimes I+\frac{1}{9}[\mathbb{I} \otimes \Lambda](\rho)
$$

which can be implemented as

$$
\widetilde{[\mathbb{I} \otimes T}](\rho)=\frac{1}{3} \Lambda_{1} \otimes \Lambda_{2}+\frac{2}{3} \mathbb{I} \otimes \sigma_{x} \sigma_{z} \Lambda_{1} \sigma_{z} \sigma_{x}
$$

where $\Lambda_{1}(\rho)=1 / 3 \sum_{i=x, y, z} \sigma_{i} \rho \sigma_{i}$, and $\Lambda_{2}(\rho)=1 / 4 \sum_{i=0, x, y, z} \sigma_{i} \rho \sigma_{i}$. Note that the map $\left.\widetilde{\mathbb{I} \otimes T}\right]$ can be implemented using only LOCC. The extension of this construction to a system of three, rather than two, qubits is trivial. All we need to consider is the map

$$
[\mathbb{I} \otimes \widetilde{\mathbb{I} \otimes T}](\rho)=\frac{1}{3} \mathbb{I} \otimes \Lambda_{1} \otimes \Lambda_{2}+\frac{2}{3} \mathbb{I} \otimes \mathbb{I} \otimes \sigma_{x} \sigma_{z} \Lambda_{1} \sigma_{z} \sigma_{x}
$$

since the composition of a CP map with identity is still a CP map, and the construction of $\widetilde{[\mathbb{I} \otimes T}]$ is independent of the existence of any additional systems. This map can again be implemented using only LOCC.

Hence, in order to check the positivity of $\rho^{T_{X}}$ with $X=A, B, C$, it is enough to implement $\mathbb{I} \otimes \widetilde{\mathbb{I} \otimes T}$ on $\rho_{\text {bound }}$, and then estimate the lowest eigenvalue of $\rho_{\text {bound }}^{\prime}=[\mathbb{I} \otimes \widetilde{\mathbb{I} \otimes T}]\left(\rho_{\text {bound }}\right)$. The estimation of the lowest eigenvalue of $\rho^{\prime}$ can be achieved bypassing full state estimation, following [26, 27].

Consider a typical set-up for single qubit interferometry, conveniently expressed in terms of quantum gates and networks: Hadamard gate, phase-shift gate, Hadamard gate, and measurement in the computational basis $\{|0\rangle,|1\rangle\}$. We modify the interferometer by inserting a controlled- $V$ operation between the Hadamard gates, where $V$ is the swap operator defined as $V|\phi\rangle_{A}|\psi\rangle_{B}=|\psi\rangle_{B}|\phi\rangle_{A}, \forall|\phi\rangle,|\psi\rangle$. The control is on the qubit and $V$ acts on the quantum state $\varrho=\rho_{A} \otimes \rho_{B}$. The interaction between the qubit and the environment $\varrho$ via the controlled- $V$ leads to a modification of the observed interference pattern by the factor $\mathrm{v} e^{i \alpha}=\operatorname{Tr}\left[V\left(\rho_{A} \otimes \rho_{B}\right)\right]=\operatorname{Tr}\left[\rho_{A} \rho_{B}\right]$. The generalization of the swap 


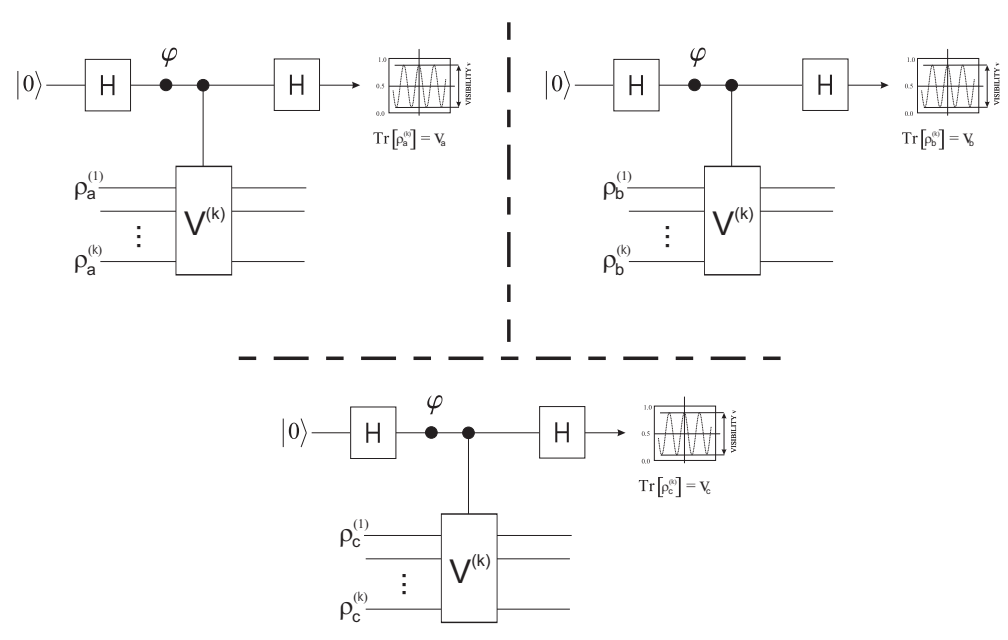

FIG. 3: Quantum network that estimates the non-linear functionals by LOCC.

operation $V$ to the shift operation $V^{(k)}$ (where $V^{(k)}\left|\phi_{1}\right\rangle\left|\phi_{2}\right\rangle \ldots\left|\phi_{k}\right\rangle=\left|\phi_{k}\right\rangle\left|\phi_{1}\right\rangle \ldots\left|\phi_{k-1}\right\rangle, \forall\left|\phi_{i}\right\rangle, i=1, \ldots, k$ ), and the choice of $\varrho=\rho^{\otimes k}$ as the input state, allows us to estimate multi-copy observables, $\operatorname{Tr}\left[\rho^{k}\right]$, of an unknown state $\rho[26]$.

Let us now extend this method to the LOCC scenario by constructing three local networks, one for Alice, one for Bob and one for Charlie, in such a way that the global network is similar to the network with the controlled-shift. Unfortunately, the global shift operation $V^{(k)}$ cannot be implemented using only LOCC. Thus, we will implement it indirectly, using the network depicted in Fig. B.

Alice, Bob and Charlie share a number of copies of the state $\rho_{A B C}$. They group them respectively into sets of $k$ elements, and run the local interferometric network on their respective thirds of the state $\varrho_{A B C}=\rho_{A B C}^{\otimes k}$. For each run of the experiment, they record and communicate their results.

The individual interference patterns Alice, Bob and Charlie record will depend only on their respective reduced density operators. Alice will observe the visibility $\mathrm{v}_{A}=\operatorname{Tr}\left[\rho_{A}^{k}\right]$, Bob will observe the visibility $\mathrm{v}_{B}=\operatorname{Tr}\left[\rho_{B}^{k}\right]$ and Charlie will observe the visibility $\mathrm{v}_{C}=\operatorname{Tr}\left[\rho_{C}^{k}\right]$. However, if they compare their individual observations, they will be able to extract information about the global density operator $\rho_{A B C}$, e.g. about

$$
\operatorname{Tr}\left[\varrho_{A B C}^{k}\right]=\operatorname{Tr}\left[\rho_{A B C}^{\otimes k}\left(V_{A}^{(k)} \otimes V_{B}^{(k)} \otimes V_{C}^{(k)}\right)\right] .
$$

This is because Alice, Bob and Charlie can estimate the probabilities $\mathrm{P}_{i j l}$ that in the measurement Alice's interfering qubit is found in state $|i\rangle_{A}$, Bob's in state $|j\rangle_{B}$ and Charlie's in state $|l\rangle_{C}$, for $i, j, l=0,1$. These probabilities can be conveniently expressed as

$$
\begin{aligned}
\mathrm{P}_{i j l}= & \frac{1}{8} \operatorname{Tr}\left[\rho_{A B C}^{\otimes k}\left(\mathbb{I}+(-1)^{i} V_{A}^{(k)}\right) \otimes\left(\mathbb{I}+(-1)^{j} V_{B}^{(k)}\right) \otimes\left(\mathbb{I}+(-1)^{l} V_{C}^{(k)}\right)\right] \\
= & \frac{1}{8}\left[1+(-1)^{i} \operatorname{Tr}\left(\rho_{A}^{k}\right)+(-1)^{j} \operatorname{Tr}\left(\rho_{B}^{k}\right)+(-1)^{l} \operatorname{Tr}\left(\rho_{C}^{k}\right)+(-1)^{i+j} \operatorname{Tr}\left(\rho_{A B}^{k}\right)\right. \\
& \left.+(-1)^{i+l} \operatorname{Tr}\left(\rho_{A C}^{k}\right)+(-1)^{j+l} \operatorname{Tr}\left(\rho_{B C}^{k}\right)+(-1)^{i+j+l} \operatorname{Tr}\left(\rho_{A B C}^{k}\right)\right] .
\end{aligned}
$$

From the latter equality it follows that

$$
\operatorname{Tr}\left(\rho_{A B C}^{k}\right)=\mathrm{P}_{000}-\mathrm{P}_{001}-\mathrm{P}_{100}-\mathrm{P}_{100}+\mathrm{P}_{011}+\mathrm{P}_{101}+\mathrm{P}_{110}-\mathrm{P}_{111} .
$$

This is equivalent to $\operatorname{Tr}\left(\rho_{A B C}^{k}\right)=\left\langle\sigma_{z} \otimes \sigma_{z} \otimes \sigma_{z}\right\rangle$, where $\sigma_{z}=|0\rangle\langle 0|-| 1\rangle\langle 1|$. Given that we are able to directly estimate $\operatorname{Tr}\left[\rho_{A B C}^{k}\right]$ for any integer value of $k$, we can estimate the spectrum of $\rho_{A B C}$ without resorting to a full state tomography. In our case $\rho_{A B C} \equiv \rho_{\text {bound }}$ and we need to estimate seven parameters, $\operatorname{Tr} \rho_{\text {bound }}^{j}$ with $j=2,3, \ldots, 8$. Together with $\operatorname{Tr} \rho_{\text {bound }}=1$ they suffice to determine the eigenvalues of $\rho_{\text {bound }}[26]$.

Even though the SPA option requires the estimation of just seven parameters of $\rho_{\text {bound }}$, it has two potential experimental difficulties, the first one being the feasibility of implementing the SPA, and the second one the feasibility of implementing the quantum networks involving $C-V$ gates.

Finally, we have the option of directly estimating the non-linear functionals $\operatorname{Tr}\left[\left(\rho^{T_{X}}\right)^{k}\right]$ with $k=1,2,3, \ldots$ and $X=A, B, C$, following [18]. This scheme is a modification of the scheme presented in [26], and can be also implemented 
using only LOCC [27]. The main difference between the quantum network of [18], when compared with 26], is that the $C-V^{(k)}$ gates acting on the different subsystems do not all shift in the same direction, that is, all but one will shift in the direction

$$
V^{(k)}\left|\alpha_{1}\right\rangle\left|\alpha_{2}\right\rangle\left|\alpha_{3}\right\rangle \ldots\left|\alpha_{k}\right\rangle=\left|\alpha_{k}\right\rangle\left|\alpha_{1}\right\rangle\left|\alpha_{2}\right\rangle \ldots\left|\alpha_{k-1}\right\rangle
$$

while the remaining subsystem will shift in the opposite direction

$$
V^{-1(k)}\left|\alpha_{1}\right\rangle\left|\alpha_{2}\right\rangle\left|\alpha_{3}\right\rangle \ldots\left|\alpha_{k}\right\rangle=\left|\alpha_{2}\right\rangle\left|\alpha_{3}\right\rangle \ldots\left|\alpha_{k}\right\rangle\left|\alpha_{1}\right\rangle
$$

In fact, the subsystem with respect to which we want to partially transpose our density operator, will be the subsystem shifted in the opposite direction. The advantage of this option, when compared to the SPA one, is that we do not have to implement any map on $\rho_{\text {bound }}$ before estimating the relevant non-linear functionals. Also, the quantum network used in both schemes has the same level of experimental difficulty.

\section{GENERATION OF THE DÜR-CIRAC-TARRACH STATES}

Another interesting family of states of three qubits, bound entagled in a certain parameter range, was introduced in 7]. In this section we will show how to produce it experimentally, with a method similar to the one described above. Using the notation from [7], this family is given by:

$$
\rho_{\mathrm{DCT}}=\sum_{\sigma= \pm} \lambda_{0}^{\sigma}\left|\Psi_{0}^{\sigma}\right\rangle\left\langle\Psi_{0}^{\sigma}\right|+\sum_{k=01,10,11} \lambda_{k}\left(\left|\Psi_{k}^{+}\right\rangle\left\langle\Psi_{k}^{+}|+| \Psi_{k}^{-}\right\rangle\left\langle\Psi_{k}^{-}\right|\right) .
$$

Here $\left|\Psi_{k}^{ \pm}\right\rangle=\frac{1}{\sqrt{2}}\left(\left|k_{1} k_{2} 0\right\rangle \pm\left|\bar{k}_{1} \bar{k}_{2} 1\right\rangle\right)$, where $k_{1}$ and $k_{2}$ are the binary digits of $k$, and $\bar{k}_{i}$ denotes the flipped $k_{i}$. (Note that the state $\left|\Psi_{0}^{+}\right\rangle$in this notation corresponds to $|G H Z\rangle$ from above.) The normalization condition reads $\lambda_{0}^{+}+\lambda_{0}^{-}+2\left(\lambda_{01}+\lambda_{10}+\lambda_{11}\right)=1$. With the definitions $\Delta \equiv \lambda_{0}^{+}-\lambda_{0}^{-} \geq 0$ and

$$
s_{k} \equiv\left\{\begin{array}{l}
1 \text { if } \lambda_{k}<\Delta / 2 \\
0 \text { if } \lambda_{k} \geq \Delta / 2
\end{array}\right.
$$

the following properties of the partial transposes hold [7]:

$$
s_{01}=0 \Leftrightarrow \rho^{T_{B}} \geq 0, \quad s_{10}=0 \Leftrightarrow \rho^{T_{A}} \geq 0, \quad s_{11}=0 \Leftrightarrow \rho^{T_{C}} \geq 0 .
$$

A singlet state between two of the parties can be distilled iff the partial transposes with respect to the two parties are negative. For the following choice of the parameters

$$
\lambda_{0}^{+}=\frac{1}{3} ; \quad \lambda_{0}^{-}=\lambda_{10}=0 ; \quad \lambda_{01}=\lambda_{11}=\frac{1}{6}
$$

the corresponding state is inseparable with respect to the splitting $A-(B C)$ but separable with respect to the other two splittings. Hence no singlet can be distilled between any of the parties and the state is bound entangled. However, when it is mixed with two states that are obtained by cyclic permutation of the parties it turns out that the mixture is inseparable with respect to any partition [28]. These properties were used recently to show that bound information exists and can be activated [12].

Let us sketch how the states of Eq. (28) could be prepared with our scheme. The density matrix is given by

$$
\rho_{\mathrm{DCT}}=\left(\begin{array}{cccccccc}
\frac{\lambda_{0}^{+}+\lambda_{0}^{-}}{2} & 0 & 0 & 0 & 0 & 0 & 0 & \frac{\lambda_{0}^{+}-\lambda_{0}^{-}}{2} \\
0 & \lambda_{11} & 0 & 0 & 0 & 0 & 0 & 0 \\
0 & 0 & \lambda_{01} & 0 & 0 & 0 & 0 & 0 \\
0 & 0 & 0 & \lambda_{10} & 0 & 0 & 0 & 0 \\
0 & 0 & 0 & 0 & \lambda_{10} & 0 & 0 & 0 \\
0 & 0 & 0 & 0 & 0 & \lambda_{01} & 0 & 0 \\
0 & 0 & 0 & 0 & 0 & 0 & \lambda_{11} & 0 \\
\frac{\lambda_{0}^{+}-\lambda_{0}^{-}}{2} & 0 & 0 & 0 & 0 & 0 & 0 & \frac{\lambda_{0}^{+}+\lambda_{0}^{-}}{2}
\end{array}\right) .
$$


We start again with the state $|000\rangle$ and produce the pure state

$$
\left|\psi_{\mathrm{DCT}}\right\rangle=\frac{\gamma}{\sqrt{2}}(|000\rangle+|100\rangle)+\sqrt{\lambda_{01}}(|010\rangle+|110\rangle)+\sqrt{\lambda_{10}}(|011\rangle+|111\rangle)+\sqrt{\lambda_{11}}(|001\rangle+|101\rangle),
$$

where $\gamma=\sqrt{\lambda_{0}^{+}+\lambda_{0}^{-}}$.

The state in Eq. (33) is reached as follows: Start by a local rotation and a CNOT gate

$$
|000\rangle \stackrel{\mathrm{LU}_{\zeta}}{\longrightarrow}|0\rangle\left(\alpha_{+}|0\rangle+\alpha_{-}|1\rangle\right)|0\rangle \stackrel{\mathrm{CNOT}_{(2,3)}}{\longrightarrow}|0\rangle\left(\alpha_{+}|00\rangle+\alpha_{-}|11\rangle\right) \quad \text { where } \quad \mathrm{LU}_{1}=\mathbb{I} \otimes\left(\begin{array}{cc}
\alpha_{+} & \alpha_{-} \\
\alpha_{-} & -\alpha_{+}
\end{array}\right) \otimes \mathbb{I} .
$$

By proper choice of the coefficients $\alpha_{ \pm}$we can then reach $\left|\psi_{\mathrm{DCT}}\right\rangle$ with 3 local unitaries described below as follows

$$
\stackrel{\mathrm{LU}_{2}}{\longrightarrow}|0\rangle\left(\gamma|00\rangle+\sqrt{2 \lambda_{01}}|10\rangle+\sqrt{2 \lambda_{10}}|11\rangle+\sqrt{2 \lambda_{11}}|01\rangle\right) \stackrel{\mathrm{LU}_{3}}{\longrightarrow}\left|\psi_{\mathrm{DCT}}\right\rangle .
$$

Hence we have to choose the coefficients and the local unitaries $\mathrm{LU}_{2}$ such that

$$
\left.\alpha_{+}|00\rangle+\alpha_{-}|11\rangle \stackrel{\mathrm{LU}_{2}}{\longrightarrow} \alpha_{+}|\phi\rangle|\psi\rangle+\alpha_{-}\left|\phi^{\perp}\right\rangle\left|\psi^{\perp}\right\rangle=\gamma|00\rangle+\sqrt{2 \lambda_{01}}|10\rangle+\sqrt{2 \lambda_{10}}|11\rangle+\sqrt{2 \lambda_{11}}|01\rangle\right),
$$

i.e. we have to find the Schmidt decomposition of the state on the RHS of the last equation. This state has the decomposition

$$
|\varphi\rangle=\sum_{i j} C_{i j}|i j\rangle \quad \text { with } \quad C=\left(\begin{array}{cc}
\gamma & \sqrt{2 \lambda_{11}} \\
\sqrt{2 \lambda_{01}} & \sqrt{2 \lambda_{10}}
\end{array}\right) .
$$

The Schmidt coefficients are the positive square roots of the eigenvalues of $C^{T} C$, namely

$$
\alpha_{ \pm}^{2}=\frac{1}{2}\left(1 \pm \sqrt{1-4\left[\left(\gamma^{2}+2 \lambda_{01}\right)\left(2 \lambda_{10}+2 \lambda_{11}\right)-\left(\gamma \sqrt{2 \lambda_{11}}+2 \sqrt{\lambda_{01} \lambda_{10}}\right)^{2}\right]}\right)
$$

Then the rotation is given by $\mathrm{LU}_{2}=\mathbb{I} \otimes V_{2} \otimes U_{2}, U_{2}=(|u+\rangle,|u-\rangle)$ and $V_{2}=(|v+\rangle,|v-\rangle)$. The vectors $|u \pm\rangle$ can be obtained from $\left(C^{T} C-\alpha_{ \pm} \mathbb{I}\right)|u \pm\rangle=0$ and the vectors $|v \pm\rangle$ from $\left(C C^{T}-\alpha_{ \pm} \mathbb{I}\right)|v \pm\rangle=0$. The last local unitary is given by $\mathrm{LU}_{3}=H \otimes \mathbb{I} \otimes \mathbb{I}$, where $H=\frac{1}{\sqrt{2}}\left(\begin{array}{cc}1 & 1 \\ 1 & -1\end{array}\right)$, the Hadamard gate.

Now we add again three ancilla qubits in the state $|000\rangle$, and by using three CNOT gates the first three qubits are copied. This yields the state

$$
\begin{aligned}
\left|\psi_{\mathrm{DCT}}\right\rangle|000\rangle \stackrel{3 \mathrm{CNOT}^{\prime} \mathrm{s}}{\longrightarrow} & \left(\frac{\gamma}{\sqrt{2}}\left(|000\rangle^{\otimes 2}+|100\rangle^{\otimes 2}\right)+\sqrt{\lambda_{01}}\left(|010\rangle^{\otimes 2}+|110\rangle^{\otimes 2}\right)\right. \\
& \left.+\sqrt{\lambda_{10}}\left(|011\rangle^{\otimes 2}+|111\rangle^{\otimes 2}\right)+\sqrt{\lambda_{11}}\left(|001\rangle^{\otimes 2}+|101\rangle^{\otimes 2}\right)\right) .
\end{aligned}
$$

Then we apply the unitary

$$
U=\frac{1}{\gamma}\left(\begin{array}{cc}
\sqrt{\lambda_{0}^{-}} & \sqrt{\lambda_{0}^{+}} \\
\sqrt{\lambda_{0}^{+}} & -\sqrt{\lambda_{0}^{-}}
\end{array}\right)
$$

on qubit 4 iff the qubits 2 and 3 are in the state $|00\rangle$. A 2-controlled operation usually acts when both control qubits are in the state $|1\rangle$, but this can be changed by flipping the control qubits before and after the gate. This operations leads to the state

$$
\begin{aligned}
& \frac{1}{\sqrt{2}}|000\rangle\left(\sqrt{\lambda_{0}^{-}}|0\rangle+\sqrt{\lambda_{0}^{+}}|1\rangle\right)|00\rangle+\frac{1}{\sqrt{2}}|100\rangle\left(\sqrt{\lambda_{0}^{+}}|0\rangle-\sqrt{\lambda_{0}^{-}}|1\rangle\right)|00\rangle \\
& +\sqrt{\lambda_{01}}\left(|010\rangle^{\otimes 2}+|110\rangle^{\otimes 2}\right)+\sqrt{\lambda_{10}}\left(|011\rangle^{\otimes 2}+|111\rangle^{\otimes 2}\right)+\sqrt{\lambda_{11}}\left(|001\rangle^{\otimes 2}+|101\rangle^{\otimes 2}\right)
\end{aligned}
$$

Then a 3-Toffoli gate flips qubit 4 iff the first three qubits are in the state $|100\rangle$. Finally two CNOT gates flip qubits 2 and 3 iff the first qubits' state is $|1\rangle$. Tracing out the ancilla particles then yields $\rho_{\mathrm{DC}}$. Summarizing, the procedure 
is

$$
\begin{aligned}
& \left|\psi_{\mathrm{DCT}}\right\rangle|000\rangle{ }^{3 \mathrm{CNOT}^{\prime} \mathrm{s}^{2}{ }^{2} \mathrm{CU}} \longrightarrow{ }^{3} \text { Toffoli } \sqrt{\frac{\lambda_{0}^{-}}{2}}(|000\rangle-|100\rangle)|000\rangle+\sqrt{\frac{\lambda_{0}^{+}}{2}}(|000\rangle+|100\rangle)|100\rangle+\ldots \\
& \underset{\mathrm{CNOT}_{1,2}, \mathrm{CNOT}_{1,3}}{\longrightarrow}\left(\sqrt{\lambda_{0}^{-}}\left|G H Z^{-}\right\rangle|000\rangle+\sqrt{\lambda_{0}^{+}}|G H Z\rangle|100\rangle+\sqrt{\lambda_{01}}\left(|010\rangle^{\otimes 2}+|101\rangle|110\rangle\right)\right. \\
& \left.+\sqrt{\lambda_{10}}\left(|011\rangle^{\otimes 2}+|100\rangle|111\rangle\right)+\sqrt{\lambda_{11}}\left(|001\rangle^{\otimes 2}+|110\rangle|101\rangle\right)\right)
\end{aligned}
$$

which leads to

$$
\operatorname{Tr}_{4,5,6}\left|\psi_{\mathrm{DCT}}\right\rangle\left\langle\psi_{\mathrm{DCT}}\right|=\rho_{\mathrm{DCT}}
$$

The complete network is shown in Fig. 4 The existence of bound entanglement for the choice of parameters in Eq. (31) can be proved by showing that the state has a PPT with respect to two subsystems, but not with the third. This can be proved experimentally by applying the methods of Sec. 4.

Note that the method works for a general choice of the parameters for which the rank of the density matrix is full.

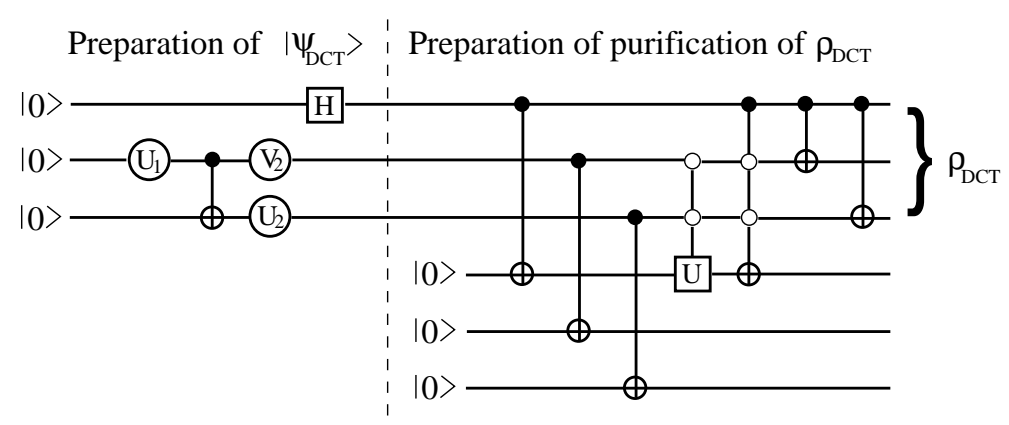

FIG. 4: The network for creating the bound entangled state given in Eq. (28). Open circles for the control bits indicate that the corresponding gate acts non-trivially on the target if the control is 0 , rather than 1 as usually (filled circles).

\section{CONCLUSIONS AND ACKNOWLEDGEMENT}

To summarize, we have presented a quantum network that generates bound entangled states of three qubits. Explicitly, we have studied the production of the two families of bound entangled states that were introduced in [14] and $[$ ]. Note that our method could be adapted in a straightforward way to the generation of other types of bound entangled states. As our networks consists of six qubits and several two-qubit gates, they go beyond present quantum information processing technology - however, it seems feasible to realize them in the not too distant future.

We also discussed different methods of testing whether the produced states generated by the network are indeed bound entangled. Namely, we suggested to detect the entanglement via a suitable witness operator, and to confirm positivity of the partial transposes by either full state estimation, or spectrum estimation of the structural physical approximation of the partial transpose, or direct estimation of some non-linear functionals.

We wish to thank A. Ekert, B. Englert, O. Gühne, D. Kaszlikowski, and M. Żukowski for discussions. We acknowledge support from Deutsche Forschungsgemeinschaft and the EU (QUPRODIS). P.H. acknowledges support from the ESF QIT short scientific visit grant. C.M.A. is supported by the Fundação para a Ciência e Tecnologia (Portugal).

[1] A. Peres, Phys. Rev. Lett. 77, 1413 (1996).

[2] M. Horodecki, P. Horodecki, and R. Horodecki, Phys. Lett. A 223, 1 (1996).

[3] M. Horodecki and P. Horodecki, Phys. Rev. A 59, 4206 (1999); M.A. Nielsen and J. Kempe, Phys. Rev. Lett. 86, 5184 (2001); O. Rudolph, J. Phys. A: Math. Gen. 33, 3951 (2000).

[4] B. Terhal, Phys. Lett. A 271, 319 (2000).

[5] M. Lewenstein, B. Kraus, J.I. Cirac, and P. Horodecki, Phys. Rev. A 62, 052310 (2000). 
[6] M. Horodecki, P. Horodecki, and R. Horodecki, Phys. Rev. Lett. 80, 5239 (1998).

[7] W. Dür, J.I. Cirac, and R. Tarrach, Phys. Rev. Lett. 83, 3562 (1999); W. Dür and J.I. Cirac, Phys. Rev. A 61, 042314 (2000).

[8] W. Dür, J. I. Cirac, M. Lewenstein, and D. Bruß, Phys. Rev. A 61, 062313 (2000); D.P. DiVincenzo, P.W. Shor, J.A. Smolin, B.M. Terhal, A.V. Thapliyal, Phys. Rev. A 61, 062312 (2000).

[9] P. Horodecki, M. Horodecki, and R. Horodecki, Phys. Rev. Lett. 82, 1056 (1999).

[10] K.G.H. Vollbrecht and M.M. Wolf, quant-ph/0201103

[11] K. Horodecki, M. Horodecki, P. Horodecki, and J. Oppenheim, quant-ph/0309110

[12] A. Acin, J. I. Cirac, and Ll. Masanes; quant-ph/0311064

[13] P. Horodecki, Phys. Lett. A 232, 333 (1997); C.H. Bennett et al., Phys. Rev. Lett. 82, 5385 (1999); D. Bruß and A. Peres, Phys. Rev. A 61, 30301 (2000).

[14] A. Acin, D. Bruß, M. Lewenstein and A. Sanpera, Phys. Rev. Lett. 87, 40401 (2001).

[15] L. Vandersypen et al, Phys. Rev. Lett. 85, 5452 (2000).

[16] Q.A. Turchette et al, Phys. Rev. Lett. 813631 (1998); S. Gulde at al., Nature 422, 408 (2003); S. Gulde et al., Nature 421, 48-50 (2003).

[17] P. Horodecki and A. Ekert, Phys. Rev. Lett. 89, 127902 (2002).

[18] H.A. Carteret, quant-ph/0309216

[19] M.A. Nielsen and I.L. Chuang, "Quantum Computation and Quantum Information", Cambridge Univ. Press, Cambridge, UK (2000).

[20] A. Barenco et al, Phys. Rev. A 52, 3457 (1995).

[21] D. Maslov and G. W. Dueck, quant-ph/0403053

[22] O. Gühne, P. Hyllus, D. Bruß, A. Ekert, M. Lewenstein, C. Macchiavello, and A. Sanpera, Phys. Rev. A 66, 062305 (2002).

[23] There is a different method presented in K. Chen and L.A. Wu, quant-ph/0306041 known to be stronger in some cases. However, in general these witnesses are more difficult to decompose locally.

[24] O. Gühne and P. Hyllus, Int. J. Theor. Phys. 42, 1001 (2003).

[25] W. Band and J. L. Park, Am. J. Phys. 47, 188 (1979); G. M. D'Ariano, Phys. Lett. A 268, 151 (2000).

[26] A.K. Ekert, C. Moura Alves, D.K.L. Oi, M. Horodecki, P. Horodecki, and L.C. Kwek, Phys. Rev. Lett. 88, 217901 (2002).

[27] C. Moura Alves et al., quant-ph/0304123

[28] W. Dür and J.I. Cirac, J. Phys. A 34, 6837 (2001). 